\title{
NOTE ON ESTIMATION OF THE NUMBER OF THE CRITICAL VALUES AT INFINITY
}

\author{
By Le Van Thanh and Mutsuo OKa
}

1. Let $f(x, y)$ be a polynomial of degree $d$ and we consider the polynomial function $f: \mathbf{C}^{2} \rightarrow \mathbf{C}$. Let $\Sigma(f)$ be the critical values. The restriction

$$
f: \mathbf{C}^{2}-f^{-1}(\Sigma) \rightarrow \mathbf{C}-\Sigma
$$

is not necessarily a locally trivial fibration. We say that $\tau \in \mathbf{C}$ is a regular value at infinıty of the function $f: \mathbf{C}^{2} \rightarrow \mathbf{C}$ if there exist positive numbers $R$ and $\varepsilon$ so that the restriction of $f, f: f^{-1}\left(D_{\varepsilon}(\tau)\right)-B_{R}^{4} \rightarrow D_{\varepsilon}(\tau)$, is a trivial fibration over the disc $D_{\varepsilon}(\tau)$ where $D_{\varepsilon}(\tau)=\{\eta \in \mathbf{C} ;|\eta-\tau| \leq \varepsilon\}$ and $B_{R}^{4}=\left\{(x, y) ;|x|^{2}+|y|^{2} \leq R\right\}$. Otherwise $\tau$ is a called a critical value at infinity. We denote the set of the critical values at infinity by $\Sigma_{\infty}$. It is known that $\Sigma_{\infty}$ is finite ([23], [2]). The purpose of this note is to give an estimation on the number of critical values at infinity. The detail will be published elsewhere ([12]).

We first consider the canonical projective compactification $\mathbf{C}^{2} \subset \mathbf{P}^{2}$. We denote the homogeneous coordinates of $\mathbf{P}^{2}$ by $X, Y, Z$ so that $x=X / Z$ and $y=Y / Z$ Let $L_{\infty}$ be the line at infinity: $L_{\infty}=\{Z=0\}$. Write

$$
f(x, y)=f_{0}+f_{1}(x, y)+\cdots+f_{d}(x, y)
$$

where $f_{i}(x, y)$ is a homogeneous polynomial of degree $i$ for $i=0, \ldots, d$. We can write

$$
f_{d}(x, y)=c x^{\nu_{0}} y^{\nu_{k+1}} \prod_{\jmath=1}^{k}\left(y-\lambda_{j} x\right)^{\nu_{j}}
$$

where $c \in \mathbf{C}^{*}$ and $\lambda_{1}, \ldots, \lambda_{k}$ are non-zero distinct numbers and we assume that $\nu_{i}>0$ for $1 \leq i \leq k$ and $\nu_{0}, \nu_{k+1} \geq 0$. Note that we have the equality

$$
\nu_{0}+\cdots+\nu_{k+1}=d
$$

Let $C_{\tau}$ be the projective curve which is the closure of the fiber $f^{-1}(\tau)$. Then $C_{\tau}$ is defined by $C_{\tau}=\left\{(X ; Y ; Z) \in \mathbf{P}^{2} ; F(X, Y, Z)-\tau Z^{d}=0\right\}$ where $F(X, Y, Z)$ is the homogeneous polynomial defined by

$$
F(X, Y, Z)=f(X / Z, Y / Z) Z^{d}=f_{0} Z^{d}+f_{1}(X, Y) Z^{d-1}+\cdots+f_{d}(X, Y)
$$

The intersection of $C_{\tau}$ and the line at infinity, $C_{\tau} \cap L_{\infty}$, is independent of $\tau \in \mathbf{C}^{2}$ and it is the base point locus of the family $\left\{C_{\tau} ; \tau \in \mathbf{C}\right\}$. Obviously we have $C_{\tau} \cap L_{\infty}=$ $\left\{Z=f_{d}(X, Y)=0\right\}$. For brevity, let $A_{\imath}=\left(\alpha_{i} ; \beta_{i} ; 0\right) \in \mathbf{P}^{2}$ for $i=0, ., k+1$ where $A_{0}=(0 ; 1 ; 0), A_{k+1}=(1 ; 0 ; 0)$ and $\beta_{i} / \alpha_{i}=\lambda_{i}$ for $1 \leq i \leq k$. Then under the assumption (1.1), $C_{0} \cap L_{\infty}=\left\{A_{2} ; \nu_{i}>0\right\}$. Note that $A_{\imath} \in C_{0} \cap L_{\infty}$ for $i=1, \ldots, k$. We consider the family of germs of a curve at $A_{\jmath}:\left\{\left(C_{\tau}, A_{\jmath}\right) ; \tau \in \mathbf{C}\right\}$. Then it is known that $\tau$ is a

Received June 30, 1993. 
regular value at infinity if and only if $\left\{\left(C_{t}, A_{\jmath}\right) ; t \in \mathbf{C}\right\}$ is a topologically stable family near $t=\tau$ for any $A_{j}$ with $\nu_{j}>0$ ([2]). This is the case if $f(x, y)-\tau$ is reduced and the local Milnor number $\mu$ of the family $\left\{\left(C_{t}, A_{j}\right) ; t \in \mathbf{C}\right\}$ is constant in a neighborhood $U$ of $\tau \in \mathbf{C}$. To study the stability of the local topological type at $A_{\jmath}$, we will use the affine polar quotient along the polar curve at infinity.

\section{Affine polar quotients and a toric compactification.}

A. Affine polar quotients.

Let $\ell(x, y)=\alpha y-\beta x$ be a linear form. The polar curve $\Gamma_{\ell}(f)$ for $f$ with respect to $\ell$ is defined by the Jacobian $\Gamma_{\ell}(f)=\left\{(x, y) \in \mathbf{C}^{2} ; J(f, \ell)(x, y)=0\right\}$ where

$$
J(f, \ell)(x, y)=\alpha \frac{\partial f}{\partial x}(x, y)+\beta \frac{\partial f}{\partial y}(x, y)=0
$$

$\Gamma_{\ell}(f)$ is an affine curve of degree $d-1$ and equal to the critical locus of the mapping $(f, \ell): \mathbf{C}^{2} \rightarrow \mathbf{C}^{2}$. Let $L_{\eta}$ be the projective line $\{\alpha Y-\beta X-\eta Z=0\}$ which is the closure of the affine line $\ell^{-1}(\eta)$. The base point of this pencil $\left\{L_{\eta} ; \eta \in \mathbf{C}\right\}$ is $B=(\alpha ; \beta ; 0)$ in the homogeneous coordinates. We say that $\ell$ is generic at infinity for the polynomial $f$ if $B \notin C_{0} \cap L_{\infty}$. This is the case if and only if $f_{d}(\alpha, \beta) \neq 0$. We assume the genericity of $\ell$ hereafter. Let $\overline{\Gamma_{\ell}(f)}$ be the projective closure of $\Gamma_{\ell}(f)$ and let $\overline{\Gamma_{\ell}(f)} \cap L_{\infty}=\left\{Q_{1}, \ldots, Q_{\delta}\right\}$. Let $\gamma$ be a local analytic irreducible component of $\overline{\Gamma_{\ell}(f)}$ at $Q_{i}$. Consider an analytic parametrization $\Phi_{\gamma}:\left(D_{\varepsilon}(0), 0\right) \rightarrow\left(\gamma, Q_{i}\right)$ in a local coordinate system in a neighborhood of $Q_{i}$. In the original affine coordinates, this can be written as $\Phi_{\gamma}(t)=\left(x_{\gamma}(t), y_{\gamma}(t)\right)$ where $x_{\gamma}(t)$ and $y_{\gamma}(t)$ are Laurent series in $t$. Consider the rational number $v_{\gamma}(f, \tau)$ defined by

$$
v_{\gamma}(f, \tau)=\frac{\operatorname{val}_{t}\left(f\left(x_{\gamma}(t), y_{\gamma}(t)\right)-\tau\right)}{\operatorname{val}_{t}\left(\ell\left(x_{\gamma}(t), y_{\gamma}(t)\right)\right)}
$$

Here $\mathrm{val}_{t}$ is the standard valuation defined by the variable $t$. It is easy to see that this number depends only on $\tau, \gamma$ and $f$ and it does not depend on the choice of the parametrization. So we call this number the affine polar quotient of the the function $f(x, y)-\tau([9],[15])$. This definition is an analogy of the local polar quotient defined in [10]. In the case of $f\left(x_{\gamma}(t), y_{\gamma}(t)\right)-\tau \equiv 0$, the valuation $\operatorname{val}_{t}\left(f\left(x_{\gamma}(t), y_{\gamma}(t)\right)-\tau\right)$ is $+\infty$ by definition. Let $p$ be a positive integer. We use the convention $+\infty / \pm p= \pm \infty$ and $-\infty$ (resp. $+\infty)$ is negative (resp. positive). Note that for the definition of the affine polar quotient, the compactification does not make any difference.

We generalize the notion of a regular value at infinity. Let $A_{\imath} \in C_{0} \cap L_{\infty}$ (so $\nu_{i}>$ 0 ) and let $\tau \in \mathbf{C}$. We say that $\tau$ is a regular value at $A_{\imath}$ for $f: \mathbf{C}^{2} \rightarrow \mathbf{C}$ if there exists an open neighborhood $U$ of $A_{\imath}$ in $\mathbf{P}^{2}$ and a positive number $\varepsilon$ such that $f$ : $U \cap f^{-1}\left(D_{\varepsilon}(\tau)\right) \rightarrow D_{\varepsilon}(\tau)$ is a trivial fibration. Here $f^{-1}\left(D_{\varepsilon}(\tau)\right) \subset \mathbf{C}^{2}$ and therefore $U \cap f^{-1}\left(D_{\varepsilon}(\tau)\right) \subset U-L_{\infty}$. Now the importance of the affine polar quotients is the following lemma:

Lemma (2.1). Assume that $\ell$ is generic. Then $\tau \in \mathbf{C}$ is a regular value at $A_{\imath}$ for $f: \mathbf{C}^{2} \rightarrow \mathbf{C}$ if the affine polar quotient $v_{\gamma}(f, \tau) \geq 0$ for any local irreducible component $\gamma$ of $\overline{\Gamma_{\ell}(f)}$ at $A_{i}$.

For the proof, we reffer to [12]. 
Corollary (2.1.1)([15]). Assume that $\ell$ us generic. Then $\tau \in \mathbf{C}$ is a regular value at infinity for the function $f: \mathbf{C}^{2} \rightarrow \mathbf{C}$ if (and only if) the affine polar quotient satisfies $v_{\gamma}(f, \tau) \geq 0$, for any local irreducible component $\gamma$ of $\overline{\Gamma_{\ell}(f)}$ at $A_{i}, i=0, \ldots, k+1$.

Note that $\operatorname{val}_{t}\left(\ell\left(x_{\gamma}(t), \underline{y}_{\gamma}(t)\right)\right)<0$ as $\left\|\left(x_{\gamma}(t), y_{\gamma}(t)\right)\right\| \rightarrow \infty$. Thus for any irreducible

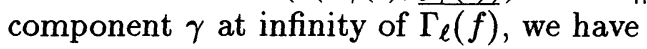

$$
v_{\gamma}(f, \tau) \geq 0 \Longleftrightarrow \operatorname{val}_{t}\left(f\left(x_{\gamma}(t), y_{\gamma}(t)\right)-\tau\right) \leq 0
$$

Lemma (2.2). Assume that $\ell$ is generic. Choose $A_{\imath}$ with $\nu_{i} \geq 2$ and let $\left(x_{\gamma}(t), y_{\gamma}(t)\right)$ be a parametrization of a local urreducible component $\gamma$ of $\overline{\Gamma_{\ell}(f)}$ at $A_{\imath}$.

(i) If $v_{\gamma}(f ; 0)>0$, then $v_{\gamma}(f ; \tau)>0$ for any any $\tau \in \mathbf{C}$.

(ii) If $v_{\gamma}(f ; 0) \leq 0$, there exısts a unique $\xi \in \mathbf{C}$ so that $v_{\gamma}(f ; \xi)<0$. For any other $\tau \neq \xi, v_{\gamma}(f ; \tau)=0$.

Proof. Assume first that $v_{\gamma}(f ; 0)>0$. Then $\operatorname{val}_{t}\left(f\left(x_{\gamma}(t), y_{\gamma}(t)\right)\right)<0$ by $(2.1 .2)$ and therefore $\operatorname{val}_{t}\left(f\left(x_{\gamma}(t), y_{\gamma}(t)\right)-\tau\right)<0$ for any $\tau$.

Assume that $v_{\gamma}(f ; 0) \leq 0$. This implies that $\operatorname{val}_{t}\left(f\left(x_{\gamma}(t), y_{\gamma}(t)\right)\right) \geq 0$. Then $\lim _{t \rightarrow 0}$ $f\left(x_{\gamma}(t), y_{\gamma}(t)\right)$ is well defined. So we denote this limit by $\xi$. Then it is obvious that $\operatorname{val}_{t}\left(f\left(x_{\gamma}(t), y_{\gamma}(t)\right)-\tau\right)=0$ for any $\tau \neq \xi$. This completes the proof.

Definition (2.3). We call that a local irreducible component $\gamma$ of $\overline{\Gamma_{\ell}(f)}$ at $A_{2}$ is stable (respectively unstable) if $v_{\gamma}(f ; 0)>0$ (resp. $\left.v_{\gamma}(f ; 0) \leq 0\right)$. We denote the set of unstable local irreducible components of $\overline{\Gamma_{\ell}(f)}$ at infinity by $\mathcal{U S}\left(\Gamma_{\ell}\right)$. Assume that $\gamma$ is a unstable local irreducible component and let $\xi$ be the complex number characterized in (ii). Considering $\xi$ as a function of $\gamma$, we write $\xi(\gamma)$. Thus we have a mapping $\xi$ : $\mathcal{U S}\left(\Gamma_{\ell}\right) \rightarrow$ C. $\xi(\gamma)$ is called the limit critıcal value of $f$ along $\gamma$.

CoRollary (2.3.1). The number of the critıcal values at infinity $\left|\Sigma_{\infty}\right|$ is equal to the cardinality of the image $\xi\left(\mathcal{U S}\left(\Gamma_{\ell}\right)\right)$. In particular, it is less than or equal to the cardinality of $\mathcal{U S}\left(\Gamma_{\ell}\right)$.

We define the projectıve degeneracy at infinıty $\nu_{\infty}^{p r}(f)$ by

$$
\nu_{\infty}^{p r}(f)=\sum_{i=0}^{k+1} \max \left(\nu_{i}-1,0\right)
$$

As the number of irreducible components of $\overline{\Gamma_{\ell}}$ at $A_{\imath}$ is less than or equal to $\nu_{i}-1$, we have the following estimation.

THEOREM (2.4). The number of critical points at infinity $\left|\Sigma_{\infty}\right|$ is less than or equal to $\nu_{\infty}^{p r}(f)$. In partıcular, $\left|\Sigma_{\infty}\right| \leq d-1$.

This estimation can be obtained using the projective compactification but it is not so good when $\nu_{0}$ or $\nu_{k+1}$ is big. It turns out that a suitable toric compactification is more convenient for our purpose. 
B. Toric Compactification of $\mathbf{C}^{2}$.

Let $f(x, y)=\sum_{(m, n)} a_{m, n} x^{m} y^{n}$ be a given polynomial of degree $d$. As we are interested in the estimation of the number of critical values at infinity, we may assume that $f(0,0) \neq 0$ by adding a constant if necessary. We consider the Newton polygon $\Delta(f)$ of $f$ which is the convex hull of the integral point $(m, n)$ such that $a_{m, n} \neq 0$. By the assumption $f(0,0) \neq 0$, we have $O \in \Delta(f)$. Let $N$ be the space of covectors. Any covector $P$ defines a linear function on $\Delta(f)$. For any integral covector $P={ }^{t}(p, q)$, let $\Delta(P ; f) \subset \Delta(f)$ be the locus where the linear function $P \mid \Delta(f)$ takes the minimal value. We denote this minimal value by $d(P ; f)$ as usual. Let $f_{P}(x, y)$ be the partial sum

$$
f_{P}(x, y):=\sum_{(m, n) \in \Delta(P ; f)} a_{m, n} x^{m} y^{n}
$$

and we call $f_{P}$ the face function of the covector $P$. The dual Newton diagram $\Gamma^{*}(f)$ is defined by the following equivalence relation in $N: P \sim Q$ if and only if $\Delta(P ; f)=\Delta(Q ; f)$. Here $\Delta(P ; f)$ is the locus where the linear function $P \mid \Delta(f)$ takes its minimal value. Let $\Sigma^{*}$ be a regular simplicial cone subdivision of $\Gamma^{*}(f)$ and let $X$ be the toric variety associated with $\Sigma^{*}$. Let $E_{1}={ }^{t}(1,0), E_{2}={ }^{t}(0,1)$. It is easy to see that $\operatorname{Cone}\left(E_{1}, E_{2}\right)$ is admissible with $\Sigma^{*}$. This is immediate from the assumption that $O \in \Delta(f)$. Thus we may assume that $\operatorname{Cone}\left(E_{1}, E_{2}\right)$ is a simplicial cone in $\Sigma^{*}$. Let $R_{1}, \ldots, R_{\mu}$ be the vertices of $\Sigma^{*}$ in the counter-clockwise orientation where $R_{1}=E_{1}, R_{2}=E_{2}$. Thus $\sigma_{\imath}:=\operatorname{Cone}\left(R_{\imath}, R_{\imath+1}\right), i=1, \ldots, \mu$ be the two-dimensional simplicial cones in $\Sigma^{*}$ where $R_{\mu+1}=R_{1}$. Here we assume $R_{1}=E_{1}, R_{2}=E_{2}, R_{\mu+1}=R_{1}$. Let $\sigma_{1}=\operatorname{Cone}\left(E_{1}, E_{2}\right)$. Recall that $X$ is a smooth compact toric variety of dimension 2 whose affine charts are $\mathbf{C}_{\sigma_{i}}^{2} ; i=1, \ldots, \mu$ and it has the canonical decomposition

$$
X=\mathbf{C}^{* 2} \coprod_{i=1}^{\mu} \widehat{E}\left(R_{i}\right)
$$

where $\widehat{E}\left(R_{\imath}\right)$ is a rational curve corresponding to the vertex $R_{i} \in \operatorname{Vertex}\left(\Sigma^{*}\right)$. The divisor $\widehat{E}\left(R_{\imath}\right)$ intersects with $\widehat{E}\left(R_{\imath-1}\right)$ and $\widehat{E}\left(R_{\imath+1}\right)$. So the dual graph of the divisors $\widehat{E}\left(R_{\imath}\right), i=1, \ldots, \mu$ makes a cycle. Taking a subdivision if necessary, we may assume that $H:={ }^{t}(-1,-1)$ in $\operatorname{Vertex}\left(\Sigma^{*}\right)$. Thus we assume that $H=R_{\theta}$ for some $3 \leq \theta \leq \mu$. The projective compactification corresponds to the smallest simplicial cone $\Sigma_{0}^{*}$ which has three vertices $\left\{E_{1}, E_{2}, H\right\}$. Let $\left(u_{i}, v_{i}\right)$ be the corresponding coordinates of the chart $\mathbf{C}_{\sigma_{i}}^{2}$. Let us consider the unimodular matrix $\sigma_{\imath}^{\prime}$ corresponding to the vertices of the cone $\sigma_{\imath}$ :

$$
\sigma_{\imath}^{\prime}=\left(\begin{array}{ll}
a_{i} & a_{i+1} \\
b_{i} & b_{i+1}
\end{array}\right)
$$

$\left(a_{i} b_{i+1}-a_{i+1} b_{i}=1\right)$. Then the original affine space is identified with the coordinate space $\mathbf{C}_{\sigma_{1}}^{2}$ with $x=u_{1}, y=v_{1}$. Recall that $\mathbf{C}_{\sigma_{i}}^{2}$ is glued with the original affine space $\mathbf{C}^{2}$ by

$$
\left\{\begin{array}{l}
x=u_{i}^{a_{2}} v_{i}^{a_{2+1}} \\
y=u_{i}^{b_{i}} v_{i}^{b_{i+1}}
\end{array}, \quad\left\{\begin{array}{l}
u_{i}=x^{b_{\imath+1}} y^{-a_{\imath+1}} \\
v_{i}=x^{-b_{\imath}} y^{a_{\imath}}
\end{array}\right.\right.
$$

We consider the curve $C=\left\{(x, y) \in \mathbf{C}^{2} ; f(x, y) \equiv 0\right\}$ in the original affine space $\mathbf{C}^{2}$ and let $\widetilde{C}$ be the closure of $C$ in $X$. The curve $\widetilde{C}$ is defined in $\mathbf{C}_{\sigma_{2}}^{2}$ by the equation 
$f_{\sigma_{i}}\left(u_{i}, v_{i}\right)=0$ where $f_{\sigma_{i}}\left(u_{i}, v_{i}\right)$ is defined by

$$
f_{\sigma_{i}}\left(u_{i}, v_{i}\right):=f\left(u_{i}^{a_{2}} v_{i}^{a_{i+1}}, u_{i}^{b_{i}} v_{i}^{b_{2+1}}\right) / u_{i}^{d\left(R_{i} ; f\right)} v_{i}^{d\left(R_{i+1} ; f\right)}
$$

In $\mathbf{C}_{\sigma_{i}}^{2}, \widehat{E}\left(R_{\imath}\right)$ is defined by $u_{i}=0$. It is easy to see that $f_{\sigma_{i}}(0,0) \neq 0$ and

$$
f_{\sigma_{i}}\left(0, v_{i}\right)=f_{R_{i}}\left(u_{i}^{a_{i}} v_{i}^{a_{i+1}}, u_{i}^{b_{i}} v_{i}^{b_{i+1}}\right) / u_{i}^{d\left(R_{i} ; f\right)} v_{i}^{d\left(R_{i+1} ; f\right)}
$$

is non-constant if and only if $\operatorname{dim} \Delta\left(R_{\imath} ; f\right) \geq 1$. Let $D_{1}, \ldots, D_{m}$ be the faces of $\Delta(f)$ in the counter-clockwise orientation so that $D_{1}, D_{m}$ contains the origin $O$. Let $P_{i}={ }^{t}\left(p_{i}, q_{i}\right)$ be the corresponding primitive integral covector of $D_{i}$. Note that each $P_{i}$ must be a vertex of $\Sigma^{*}$ and therefore we can write $P_{i}=R_{\nu_{i}}$ for some $1 \leq \nu_{i} \leq \mu$. Then we can write

$$
f_{P_{\imath}}(x, y)=\delta_{i} x^{r_{i}} y^{s_{i}} \prod_{\jmath=1}^{\ell_{i}}\left(y^{p_{i}}-\xi_{i, \jmath} x^{q_{i}}\right)^{\nu i, \jmath}
$$

where $\delta_{i} \in \mathbf{C}^{*}$ and $\xi_{i, j}, 1 \leq j \leq \ell_{i}$ are mutually distinct non-zero complex numbers. By the above consideration, $\widehat{E}\left(R_{\imath}\right) \cap C \neq \emptyset$ if and only if $i=\nu_{j}$ for some $1 \leq j \leq m$. We consider the toric coordinate chart $\sigma_{\nu_{i}}=\operatorname{Cone}\left(R_{\nu_{i}}, R_{\nu_{i}+1}\right)$. Then

$$
h_{\sigma_{i}}\left(0, v_{i}\right)=\delta_{i} \prod_{j=1}^{\ell_{i}}\left(v_{i}-\xi_{i, j}\right)^{\nu \imath, j}
$$

Thus $\widehat{E}\left(R_{\nu_{\imath}}\right) \cap \widetilde{C}$ consists of $\ell_{\imath}$ points $\left\{\left(0, \xi_{i, j}\right) ; j=1, \ldots, \ell_{i}\right\} \subset \mathbf{C}_{\sigma_{\nu_{i}}}^{2}$. Put $A_{\imath, J}:=$ $\left(0, \xi_{i, 3}\right) \in \widehat{E}\left(R_{\nu_{i}}\right) \cap \widetilde{C}$ for $1 \leq i \leq m, 1 \leq j \leq \ell_{2}$. See [20], [16], [7] for further information about the toric compactification.

Now we consider the limit of the value of the function $f$ along an irreducible component $\gamma$ of $\overline{\Gamma_{\ell}(f)}$. Let $\Phi_{\gamma}(t)$ be a parametrization of $\gamma$ in the coordinates $(x, y)$ (namely in $\left.\mathbf{C}_{\sigma_{1}}^{2}\right)$ in the neighborhood of the infinity where $x_{\gamma}(t)$ and $y_{\gamma}(t)$ are Laurent series in the variable $t$. We assume that $x_{\gamma}(t), y_{\gamma}(t) \not \equiv 0$ and write them as

$$
\left\{\begin{array}{l}
x_{\gamma}(t)=\alpha_{\gamma} t^{p_{\gamma}}+\quad \text { (higher terms) } \\
y_{\gamma}(t)=\beta_{\gamma} t^{g_{\gamma}}+\quad \text { (higher terms), } \quad t \in D_{\varepsilon}(0)
\end{array}\right.
$$

Let $Q_{\gamma}:={ }^{t}\left(p_{\gamma}, q_{\gamma}\right) \in N$ and $A_{\gamma}:=\left(\alpha_{\gamma}, \beta_{\gamma}\right)$. We assume that

$$
\min \left(p_{\gamma}, q_{\gamma}\right)<0, \quad \alpha_{\gamma}, \beta_{\gamma} \neq 0
$$

so that $x_{\gamma}(t) \not \equiv 0, y_{\gamma}(t) \not \equiv 0$ and $\left|x_{\gamma}(t)\right|^{2}+\left|y_{\gamma}(t)\right|^{2} \rightarrow \infty$. In this situation, we have

Proposition (2.9). (i) We have $\operatorname{val}_{t} f\left(x_{\gamma}(t), y_{\gamma}(t)\right) \geq d\left(Q_{\gamma} ; f\right)$ and the inequality holds if and only if $Q_{\gamma} \sim P_{i}$ and $\beta_{\gamma}^{p_{2}}-\xi_{\imath, j} x_{\gamma}^{q_{i}}=0$ for some $i, 1 \leq i \leq m$ and $j, 1 \leq j \leq \ell_{2}$.

(ii) The limit $\lim _{t \rightarrow 0} \Phi_{\gamma}(t)$ in $X$ always exists and we have

$$
\lim _{t \rightarrow 0} \Phi_{\gamma}(t)= \begin{cases}(0,0) \in \mathbf{C}_{\sigma_{\jmath}}^{2} & \text { if } Q_{\gamma} \in \operatorname{IntCone}\left(R_{\jmath}, R_{\jmath+1}\right) \\ \left(0, \alpha_{\gamma}^{-b_{\jmath}} \beta_{\gamma}^{b_{\jmath}}\right) \in \mathbf{C}_{\sigma_{\jmath}}^{2} & \text { if } Q_{\gamma}=c R_{\jmath}, \text { for some } c>0\end{cases}
$$

Here IntCone $\left(R_{\jmath}, R_{\jmath+1}\right)$ is the open cone generated by $R_{\jmath}$ and $R_{\jmath+1}$. In particular, if $Q_{\gamma} \sim P_{i}$ and $\beta_{\gamma}^{p_{i}}-\xi_{i, j} x_{\gamma}^{q_{i}}=0$ for some $i, 1 \leq i \leq m, \lim _{t \rightarrow 0} \Phi_{\gamma}(t)=\left(0, \xi_{i, j}\right) \in \mathbf{C}_{\sigma_{\nu_{i}}}^{2}$.

We refer to [12] for the further detail. Note that $d\left(Q_{\gamma} ; f\right) \leq 0$. By (i) and Lemma (2.1), we have to check the stability at $A_{\imath, \jmath}$ with $\nu_{i, \jmath} \geq 2$. 


\section{Toric estimation.}

Let $f(x, y)$ be as before. We will generalize Theorem (2.4) using the toric embedding theory. We assume for brevity that $\operatorname{dim} \Delta(f)=2$ but every argument works even in the case $\operatorname{dim} \Delta(f)=1$. Let $D_{1}, \ldots, D_{m}$ be the faces of $\Delta(f)$ in the clockwise orientation so that $D_{1}, D_{m}$ contain the origin. Let $P_{i}={ }^{t}\left(p_{i}, q_{i}\right)$ be the corresponding primitive integral covector of $D_{i}$. To get a better estimation, we first introduce the reduced polynomial $\widetilde{f}(x, y):=f(x, y)-f(0,0)$. Note that $\Delta(\widetilde{f}) \subset \Delta(f)$ but $O \notin \Delta(\tilde{f})$. We factorize $f_{P_{i}}(x, y)$ as follows.

$$
\tilde{f}_{P_{i}}(x, y)=\delta_{i} x^{r_{i}} y^{s_{i}} \prod_{j=1}^{\ell_{i}}\left(y^{p_{i}}-\xi_{i, j} x^{q_{i}}\right)^{\nu_{i, j}}
$$

Note that $f_{P_{i}}(x, y)=\tilde{f}_{P_{i}}(x, y)$ for $i=2, \ldots, m-1$. We define the following integers

$$
\begin{gathered}
\nu\left(D_{i}\right)=\sum_{i=1}^{\ell_{2}}\left(\nu_{i, j}-1\right), \quad \eta\left(D_{i}\right)=\sum_{i=1}^{\ell_{2}} \nu_{i, j} \\
\eta\left(D_{1}\right)^{\prime}=\left\{\begin{array}{ll}
\eta\left(D_{1}\right), & p_{1}<0 \\
0, & p_{1}=0
\end{array}, \quad \eta\left(D_{m}\right)^{\prime}= \begin{cases}\eta\left(D_{m}\right), & q_{m}<0 \\
0, & q_{m}=0\end{cases} \right. \\
\varepsilon_{x}(f)=s_{1}+p_{1} \sum_{j=1}^{\ell_{1}} \nu_{1, \jmath}, \\
\varepsilon(f)= \begin{cases}0, & \max \left(\varepsilon_{x}(f), \varepsilon_{y}(f)\right) \leq 1 \\
1, & \max \left(\varepsilon_{x}(f), \varepsilon_{y}(f)\right) \geq 2\end{cases}
\end{gathered}
$$

Note that $\varepsilon_{x}(f)$ (respectively $\left.\varepsilon_{y}(f)\right)$ is the $y$-coordinate (resp. $x$-coordinate) of the left side edge of $\widetilde{\Delta}_{1}=\Delta\left(P_{1} ; \widetilde{f}\right)\left(\operatorname{resp} . \widetilde{\Delta}_{m}=\Delta\left(P_{m} ; \widetilde{f}\right)\right)$.

Let us define define the toric degeneracy $\nu_{\infty}^{\text {tor }}(f)$ by

$$
\nu_{\infty}^{\text {tor }}(f)=\sum_{i=2}^{m-1} \nu\left(D_{i}\right)+\eta\left(D_{1}\right)^{\prime}+\eta\left(D_{m}\right)^{\prime}+\varepsilon(f)
$$

The toric degeneracy $\nu_{\infty}^{\text {tor }}(f)$ is smaller than the projective degeneracy $\nu_{\infty}^{p r}(f)$ in general. Now we are ready to state the main theorem.

MaIN TheOREM (3.4). The number of critical values from infinity of the function $f$ is less than or equal to $\nu_{\infty}^{\text {tor }}(f)$.

We say that $f(x, y)$ is non-degenerate on the outside boundary if $\nu\left(D_{i}\right)=0$ for any $2 \leq i \leq m-1$. Recall that $\tilde{f}$ is convenient iff $\tilde{f}(x, 0) \not \equiv 0$ and $\tilde{f}(0, y) \not \equiv 0$.

Corollary (3.4.1) ([20]). Assume that $\tilde{f}(x, y)$ is a convenient polynomial. Then $\nu_{\infty}^{\text {tor }}(f)=\sum_{\imath=2}^{m-1} \nu\left(D_{i}\right)$. In partıcular, if $\tilde{f}(x, y)$ has non-degenerate outside Newton boundaries, $f$ has no critical value from the infinity.

We give an outline of the Main theorem. For the detail, we refer to [12]. 
Let $\gamma$ be an unstable irreducible component of $\overline{\Gamma_{\ell}(f)}$ at infinity and let $\Phi_{\gamma}(t)$ be a parametrization of $\gamma$ in the coordinates $(x, y)$ where $x_{\gamma}(t)$ and $y_{\gamma}(t)$ are Laurent series in the variable $t$. We assume first that

$$
x_{\gamma}(t), y_{\gamma}(t) \not \equiv 0 \quad \text { and } \quad\left|x_{\gamma}(t)\right|^{2}+\left|y_{\gamma}(t)\right|^{2} \rightarrow \infty(t \rightarrow 0)
$$

and we expand them in Laurent series as

$$
\left\{\begin{array}{l}
x_{\gamma}(t)=a_{\gamma} t^{p_{\gamma}}+\quad \text { (higher terms) } \\
y_{\gamma}(t)=b_{\gamma} t^{q_{\gamma}}+\quad \text { (higher terms) }
\end{array}\right.
$$

The case $x_{\gamma}(t) y_{\gamma}(t) \equiv 0$ will be treated later. Let $Q_{\gamma}:={ }^{t}\left(p_{\gamma}, q_{\gamma}\right) \in N$ and $A_{\gamma}:=\left(a_{\gamma}, b_{\gamma}\right)$. By the assumption we have that

$$
A_{\gamma} \in \mathbf{C}^{* 2}, \quad \min \left(p_{\gamma}, q_{\gamma}\right)<0
$$

First we have the following Proposition:

Proposition (3.7). We have $\operatorname{val}_{t} f\left(\ell\left(x_{\gamma}(t), y_{\gamma}(t)\right)\right) \geq d\left(Q_{\gamma} ; f\right)$ and the inequality holds if and on if

$$
Q_{\gamma}=c P_{i}, \quad f_{P_{i}}\left(A_{\gamma}\right)=0 \text { for some } c>0 \quad \text { and } 1 \leq i \leq m .
$$

Recall that $\Gamma_{\ell}(f)$ is defined by $\Gamma_{\ell}(f)=\left\{(x, y) \in \mathbf{C}^{2} ; J(x, y)=0\right\}$ where

$$
J(x, y)=\alpha \frac{\partial f}{\partial x}(x, y)+\beta \frac{\partial f}{\partial y}(x, y)=\alpha \frac{\partial \tilde{f}}{\partial x}(x, y)+\beta \frac{\partial \tilde{f}}{\partial y}(x, y)=0
$$

First we observe that the Newton boundary $\Delta(J)$ is slightly different from $\Delta(\tilde{f})$ but the following is enough for our purpose.

$$
J_{Q_{\gamma}}(x, y)= \begin{cases}\alpha \frac{\partial \tilde{f}_{Q_{\gamma}}}{\partial x}(x, y)+\beta \frac{\partial \tilde{f}_{Q_{\gamma}}}{\partial y}(x, y) & p_{\gamma}=q_{\gamma} \\ \alpha \frac{\partial \tilde{f}_{Q_{\gamma}}}{\partial x}(x, y) & p_{\gamma}>q_{\gamma}, \tilde{f}_{Q_{\gamma}}(x, y) \neq \tilde{f}_{Q_{\gamma}}(0, y) \\ \beta \frac{\partial \tilde{f}_{Q_{\gamma}}}{\partial y}(x, y) & p_{\gamma}<q_{\gamma}, \tilde{f}_{Q_{\gamma}}(x, y) \neq \tilde{f}_{Q_{\gamma}}(x, 0)\end{cases}
$$

We divide the situation in two cases.

CASE I. $d\left(Q_{\gamma} ; f\right)<0 . \quad$ CASE II. $d\left(Q_{\gamma} ; f\right)=0$.

We first consider the case:

CASE I. $d\left(Q_{\gamma} ; f\right)<0$. We assume that $\gamma$ is an unstable irreducible component of $\overline{\Gamma_{\ell}(f)}$ at infinity. Then by Proposition (3.7), we must have $Q_{\gamma}=c P_{i}$ with $2 \leq i \leq$ $m-1$. We call the face $D_{2}, \ldots, D_{m-1}$ the outside faces of $\Delta(f)$. We ask how many such components are possible for a fixed $i$. By an easy computation, we can see that the multiplicity of $y^{p_{i}}-\xi_{i, \jmath} x^{q_{i}}$ in the factorization of $J_{P_{i}}(x, y)$ is exactly $\nu_{i, \jmath}-1$. Thus by the argument in the previous section, the local equation of $\overline{\Gamma_{\ell}(f)}$ in the toric coordinate 
$\operatorname{chart} \mathbf{C}_{\sigma_{\nu_{i}}}^{2}$ is of the form

$$
\delta_{i} \eta\left(v_{\nu_{i}}\right)\left\{\prod_{\jmath=1}^{\ell_{i}}\left(v_{\nu_{i}}-\xi_{i, J}\right)^{\nu_{i, j}-1}+u_{\nu_{i}} g\left(u_{\nu_{i}}, v_{\nu_{i}}\right)\right\}=0
$$

where $\delta_{i} \neq 0, \eta\left(v_{\nu_{i}}\right)$ is a polynomial with $\eta\left(\xi_{i, j}\right) \neq 0$ for any $j=1, \ldots, \ell_{2}$. (Recall that $P_{i}=R_{\nu_{i}}$ and $\sigma_{\nu_{i}}=\operatorname{Cone}\left(R_{\nu_{i}}, R_{\nu_{i}+1}\right)$.) Let $A_{\imath, j}=\left(0, \xi_{i, j}\right) \in \mathbf{C}_{\sigma_{\nu_{i}}}^{2}$. Thus by an easy argument, we have

Proposition (3.9). The number of local irreducible components $\gamma$ at infinity of $\overline{\Gamma_{\ell}(f)}$ such that $\lim _{t \rightarrow 0}\left(x_{\gamma}(t), y(\gamma(t))\right)=A_{i, j}$ is at most $\nu_{i, j}-1$ for any $2 \leq i \leq$ $m-1$. Thus the number of the unstable irreducible components $\gamma$ such that the limit $\lim _{t \rightarrow 0}\left(x_{\gamma}(t), y(\gamma(t))\right)$ intersect with the divisor $\widehat{E}\left(P_{i}\right)$ is bounded by $\nu\left(D_{i}\right)$.

Now we consider the second case: $d\left(Q_{\gamma} ; f\right)=0$. Then it is clear that $d\left(Q_{\gamma} ; \tilde{f}\right) \geq 0$. We divide Case II into two subcases.

$$
\text { CASE II-1. } d\left(Q_{\gamma} ; \tilde{f}\right)=0 . \quad \text { CASE II- } 2 . d\left(Q_{\gamma} ; \tilde{f}\right)>0
$$

Recall that $D_{1}$ and $D_{m}$ are the face which contains the origin $O$. Let $\widetilde{D_{1}}=\Delta\left(P_{1} ; \widetilde{f}\right)$ and $\widetilde{D_{m}}=\Delta\left(P_{m} ; \widetilde{f}\right)$. We call $D_{1}$ and $D_{m}$ (respectively $\widetilde{D_{1}}$ and $\left.\widetilde{D_{m}}\right)$ the right and left conıcal faces of $f(x, y)$ (respectively of $\widetilde{f}(x, y)$ ). Note that $\widetilde{D_{i}} \subset D_{i}$ and $\widetilde{D_{i}}$ might be a vertex for $i=1, m$. $\widetilde{D_{1}}, \widetilde{D_{m}}$ are called bad faces in [14]. It is more convenient to consider the factorization of $\widetilde{f}_{P_{1}}(x, y)$ :

$$
\begin{gathered}
\tilde{f}_{P_{1}}(x, y)=\delta_{1}\left(x^{q_{1}} y^{-p_{1}}\right)^{e_{1}} \prod_{j=1}^{\ell_{1}}\left(1-\xi_{1, j} x^{q_{1}} y^{-p_{1}}\right)^{\nu_{1, j}}, \quad e_{1}>0 \\
\tilde{f}_{P_{m}}(x, y)=\delta_{m}\left(x^{-q_{m}} y^{p_{m}}\right)^{e_{m}} \prod_{j=1}^{\ell_{m}}\left(x^{-q_{m}} y^{p_{m}}-\xi_{m, \jmath}\right)^{\nu_{m, J}}, \quad e_{m}>0
\end{gathered}
$$

Comparing with (3.1), we have

$$
\begin{aligned}
& r_{1}=q_{1} e_{1}, \quad s_{1}+p_{1} \sum_{\jmath=1}^{\ell_{1}} \nu_{1, \jmath}=-p_{1} e_{1} \\
& r_{m}+q_{m} \sum_{\jmath=1}^{\ell_{m}} \nu_{m, \jmath}=-q_{m} e_{m}, \quad s_{m}=p_{m} e_{m}
\end{aligned}
$$

Now we consider Case II-1 first. In this case, we must have either $Q_{\gamma}=c P_{1}$ or $Q_{\gamma}=c P_{m}$ for some $c>0$. Let us consider the case $Q_{\gamma}=c P_{1}$ for instance. By the assumption $\min \left(p_{i}, q_{i}\right)<0$ and $\operatorname{dim}(\Delta(f))=2$, we must have $p_{1}<0<q_{1}$ if such a $\gamma$ exists. Now we assert

LEMma (3.11). The number of the local irreducible components of $\overline{\Gamma_{\ell}(f)}$ of type Case II-1 with $Q_{\gamma}=c P_{1}, c>0$ (respectively $Q_{\gamma}=c P_{m}, c>0$ ) is less than or equal to $\eta\left(D_{1}\right)^{\prime}$ (respectıvely $\left.\eta\left(D_{m}\right)^{\prime}\right)$. They are all unstable. 
See [12] for the proof. Now we consider the last case:

CASE II-2. $d\left(Q_{\gamma} ; f\right)=0$ and $d\left(Q_{\gamma} ; \tilde{f}\right)>0$.

This is the case if and only if $\Delta\left(Q_{\gamma} ; f\right)=\{O\}$ and $p_{\gamma} q_{\gamma}<0$. So we assume for example

$$
p_{\gamma}<0<q_{\gamma}
$$

By the assumption $d\left(Q_{\gamma} ; \tilde{f}\right)>0$, we have that $\tilde{f}_{Q_{\gamma}}(x, 0) \equiv 0$. (If $\tilde{f}_{Q_{\gamma}}(x, 0) \not \equiv 0$, we get a contradiction: $d\left(Q_{\gamma} ; \widetilde{f}\right)<0$.) and $\tilde{f}_{Q_{\gamma}}(x, y) \neq \tilde{f}_{Q_{\gamma}}(x, 0)$. Thus by $(3.8) J_{Q_{\gamma}}(x, y)=$ $\frac{\partial f_{Q_{\gamma}}}{\partial y}(x, y)$. The assumption $\Delta\left(Q_{\gamma} ; f\right)=\{O\}$ implies that $p_{1}<0<q_{1}$ and

$$
\operatorname{det}\left(Q_{\gamma}, P_{1}\right)>0
$$

We consider the equality $J\left(x_{\gamma}(t), y_{\gamma}(t)\right) \equiv 0$. The leading term of this equality gives the following necessary condition is that

$$
J_{Q_{\gamma}}\left(A_{\gamma}\right)=\frac{\partial f_{Q_{\gamma}}}{\partial y}\left(A_{\gamma}\right)=0
$$

By (3.14), we must have

$$
\operatorname{dim} \Delta\left(Q_{\gamma} ; \tilde{f}\right)=1
$$

Such a face $\Delta\left(Q_{\gamma} ; \tilde{f}\right)$ is called an insıde face with mixed weight vector of $\tilde{f}(x, y)$. Geometrically the supporting line of such a face separates the Newton polygon $\Delta(\tilde{f})$ and the origin $O$. We consider the right conical face $\widetilde{D_{1}}$. By the expression (3.1) or (3.10), the left edge of $\widetilde{D_{1}}$ is $R:=\left(r_{1}, s_{1}+p_{1} \sum_{\jmath=1}^{\ell_{1}} \nu_{1, \jmath}\right)=\left(q_{1} e_{1},-p_{1} e_{1}\right)$. This gives a vertex $\left(q_{1} e_{1},-p_{1} e_{1}-1\right)$ of the Newton polygon $\Delta(J)$ by the differential in $y$.

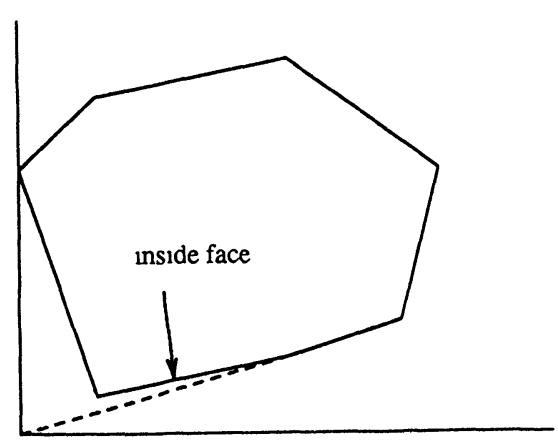

Figure (3.17.A)

If $-p_{1} e_{1}=1$, it is easy to see that there exists no inside face of mixed weight $Q_{\gamma}$ with $p_{\gamma}<0<q_{\gamma}$. Therefore we assume

$$
-p_{1} e_{1} \geq 2
$$

In this case, it is not necessary to count the number of such local irreducible components. In fact, we have 
Proposition (3.17). Each local irreducible components $\gamma$ of $\overline{\Gamma_{\ell}(f)}$ of Case II-2 gives the limit critical value $-f(0,0)$.

Proof. By the assumption, we have

$$
f\left(x_{\gamma}(t), y_{\gamma}(t)\right)=f(0,0)+(\text { higher terms) }
$$

Thus the assertion is trivial.

Until now, we have assumed that $x_{\gamma}(t), y_{\gamma}(t) \not \equiv 0$. Now we consider the exceptional case that $x_{\gamma}(t) \equiv 0$ or $y_{\gamma}(t) \equiv 0$. Assume for example

$$
\gamma: x_{\gamma}(t)=1 / t, \quad y_{\gamma}(t) \equiv 0
$$

This implies that $y$ divides $J(x, y)$. By the above argument, it is necessary that $-p_{1} e_{1} \geq$ 2. In this case, we can see that $f(x, 0) \equiv f(0,0)$. Thus if this is the case, $\operatorname{val}_{t} f\left(x_{\gamma}(t)\right.$, $\left.y_{\gamma}(t)\right)=0$ and $\gamma$ is unstable and the corresponding limit critical value is again $-f(0,0)$. Now summerizing the above argument, we have

Proposition (3.18). Assume that $-p_{1} e_{1} \geq 2$ in (3.10.1) (respectively $-q_{m} e_{m} \geq 2$ in (3.10.2)). Then either there exists an unstable local irreducible component of $\overline{\Gamma_{\ell}(f)}$ of type Case II-2 with $p_{\gamma}<0<q_{\gamma}\left(\right.$ resp. $\left.q_{\gamma}<0<p_{\gamma}\right)$, or $y=0$ (resp. $x=0$ ) is a (global) component of $\overline{\Gamma_{\ell}(f)}$. In any case, the possible limit critıcal value $\imath s-f(0,0)$.

Now we give several examples.

Example (3.19). (A) Let $\tilde{f}(x, y)=y^{2 n}+x^{3 n} y^{n}(x+y)^{n}+x^{4} y$. Then $\Delta(\widetilde{f})$ has four faces. In this example, $d=5 n$ and $\tilde{f}_{5 n}=x^{3 n} y^{n}(x+y)^{n}$, and the projective degeneracy at infinity $\nu_{\infty}^{p r}(f)=5 n-3$. On the other hand, $\eta\left(D_{l}\right)^{\prime}=n-1, \nu\left(\Delta_{2}\right)=n-1$ and $\nu\left(D_{3}\right)=0, \eta\left(D_{4}\right)^{\prime}=0$ and $\varepsilon(f)=0$. Thus we have $\nu_{\infty}^{\text {tor }}(f)=2 n-2$.

(B) Let $\tilde{f}(x, y)=x^{4} y^{4}+x y^{3}+x^{3} y^{2}+x y$. In this example, we have $\nu\left(\Delta_{2}\right)=$ $\nu\left(\Delta_{3}\right)=0, \eta\left(D_{1}\right)^{\prime}=\eta\left(D_{4}\right)^{\prime}=0$ and $\varepsilon(f)=1$ and $\nu_{\infty}^{\text {tor }}(f)=1$. In fact, 0 is the only critical point of $\tilde{f}$ from the infinity.

(C) Let $\widetilde{f}(x, y)=x+c_{2} x^{2}+\cdots+c_{n} x^{n}+x^{m} y$. Then $\Delta(\widetilde{f})$ has three faces and $\nu_{\infty}^{\text {tor }}(\widetilde{f})=1$. In fact, $\tilde{f}$ has one critical value 0 from the infinity. This polynomial has no critical point ([19]).

\section{REFERENCE}

[1] S.A. Broughton, On the topology of polynomıal hypersurfaces, Proceedings of Symposia in Pure Mathematics, 40, AMS, 1983, pp. 167-178.

[2] H.V. Hà et D.T. Lê, Sur la topologıe des polynôme complexes, Acta Math. Vietnamica 9, n.1 (1984), pp. 21-32.

[3] H.V. Hà et L.A. Nguyen, Le comportement géométrıque à l'infinı des polynômes de deux varıables, C.R. Acad. Sci. Paris t. 309 (1989), pp. 183-186.

[4] H.V. Hà, Nombres de Lojasıewıcz et singularıtés à l'infini des polynômes de deux varıables complexes, C.R. Acad. Sci. Paris t. 311 (1990), pp. 429-432.

[5] A.G. Khovanskii, Newton polyhedra and toral varieties, Funkts. Anal. Prilozhen. 11, No. 4 (1977), pp. 56-67. 
[6] A.G. Kouchnirenko, Polyèdres de Newton et Nombres de Milnor, Inventiones Math. 32 (1976), pp. 1-32.

[ 7 ] G. Kempf, F. Knudsen, D. Mumford and B. Saint-Donat, Torosdal Embeddings, vol. 339, Lecture Notes in Math., Springer, Berlin-Heidelberg-New York, 1973.

[8] D.T. Lê, Sur un critére d'equısıngularité, C.R. Acad. Sci. Paris, Ser. A-B 272 (1971), pp. 138140.

[9] V.T. Le, Affine polar quotients of algebraic plane curve, Acta. Math. Viet. 17 (1992), pp. 95-102.

[10] D.T. Lê, F. Michel and C. Weber, Courbes polaıres et topologıe des courbes planes, Ann. Sc. Ec. Norm. Sup. $4^{e}$ Series 24 (1991), pp. 141-169.

[11] D.T. Lê and M. Oka, On the Resolution Complexity of Plane Curves, preprint.

[12] V.T. Le and M. Oka, Estimation of the Number of the Critical Values at Infinity of a Polynomial Function $f: \mathbf{C}^{2} \rightarrow \mathbf{C}$, Titech-Math 07-93.

[13] J. Milnor, Singular Points of Complex Hypersurface, Annals Math. Studies, vol. 61, Princeton Univ. Press, Princeton, 1968.

[14] A. Némethi and A. Zaharia, On the bifurcation set of a polynomial function and Newton boundary, Publ. RIMS. Kyoto Univ. 26 (1990), pp. 681-689.

[15] W.D. Neumann and V.T. Le, On irregular links at infinity of algebraic plane curves, Math. Annalen 295 (1993), pp. 239-244.

[16] T. Oda, Convex Bodies and Algebraic Geometry, Springer, Berlin-Heidelberg-New York, 1987.

[17] M. Oka, On the topology of the Newton boundary II, J. Math. Soc. Japan 32 (1980), pp. 65-92.

[18] - On the topology of the Newton boundary III, J. Math. Soc. Japan 34 (1982), pp. 541-549.

[19] - On the boundary obstructions to the Jacobıcan problem, Kodai Math. J. 6 (1983), pp. 419-433.

[20] - On the topology of full non-degenerate complete intersection variety, Nagoya Math. J. 121 (1991), pp. 137-148.

[21] - Geometry of plane curves via torozdal resolution, to appear in Proceeding of Algebraic Geometry, Ladabida, 1991.

[22] _ A Lefshetz type theorem in a torus, to appear in Proceeding of College of Singularity at Trieste 1991.

[23] J.P. Verdier, Stratifications de Whitney et théorème de Bertinı-Sard, Inventiones Math. 36 (1976), pp. 295-312.

[24] O. Zariski, Studies ın equısıngularity, I, Amer. J. Math. 31 (1965), pp. 507-537.

\section{LE Van ThanH}

INSTITUTE OF MATHEMATICS

P.O. Box 631 Bo Hô, 10000 HaNOI

MUTSUO OKA

Department of Math.

TOKYO INST. OF TECH.

Oh-OKayama, Meguro-KU, TOKyo 152 